\title{
Pitiriasis rotunda en Colombia
}

\author{
Pityriasis rotunda in Colombia
}

\author{
Giovanni Manrique ${ }^{1}$, Liliana Herrera'1, Isabel Bustamante'2, María Fernanda Ordóñez² \\ 1. Médico dermatólogo, Universidad Militar Nueva Granada, Hospital Militar Central, Bogotá, D.C., Colombia \\ 2. Médica, residente de Dermatología, Universidad Militar Nueva Granada. Hospital Militar Central, Bogotá, D.C., Colombia
}

\section{RESUMEN}

La pitiriasis rotunda es una rara alteración de la queratinización. Se caracteriza por parches o placas, hiperpigmentadas o hipopigmentadas, con descamación ictiosiforme, que tienen forma de círculos geométricamente perfectos y bien definidos, frecuentemente localizadas en el tronco o en los miembros superiores. Esta condición fue descrita en Japón por Toyama en 1906, y ha sido llamada pitiriasis circinada y pseudoictiosis adquirida. La etiología aún es desconocida. La mayoría de autores creen que es una forma de ictiosis adquirida, de ictiosis congénita tardía o una manifestación cutánea de enfermedad sistémica. Hay dos tipos de presentación. El tipo I se presenta en mayores de 60 años, de raza negra o asiáticos, usualmente asociado a neoplasia maligna o enfermedad sistémica. El tipo II se presenta en pacientes blancos y jóvenes, con asociación familiar.

Se presenta el caso de un hombre de 75 años de raza negra, con manifestaciones clínicas e histopatológicas de pitiriasis rotunda, cuyos estudios de extensión para enfermedad sistémica han sido negativos.

PALABRAS CLAVE: pitiriasis, ictiosis

\section{ABSTRACT}

Pityriasis rotunda is a rare cutaneous of the keratinization disorder. It is characterized by hyper or hypopigmented, geometrically perfect circular sharply defined patches or plaques with dry ichthyosiform scaling. Lesions are frecuently distributed over the trunk and upper extremities. This condition was described in Japan by Toyoma in 1906, and had been previously called pityriasis circinata and acquired pseudoichthyosis. The etiology remains unknown; most authors believe that it is a form of acquired ichthyosis, a delayed presentation of congenital ichthyosis, or a cutaneous manifestation of systemic disease. There are two forms of presentation: Type I: in black or Asian people older than 60 years, often seen in association with malignancies or systemic diseases and type II: in younger and white people with familiar association. We present a 75 years-old black man with clinical and histopathological findings of pitiriasis rotunda with complementary studies still without alterations.

KEYWORDS: Pytiriasis, ichthyosis
Correspondencia:

María Fernanda Ordóñez,

Email:

mfordonezr@gmail.com

Recibido: 8 de mayo 2016

Aceptado: 16 de octubre de 2016

No se reportan conflictos de interés. 


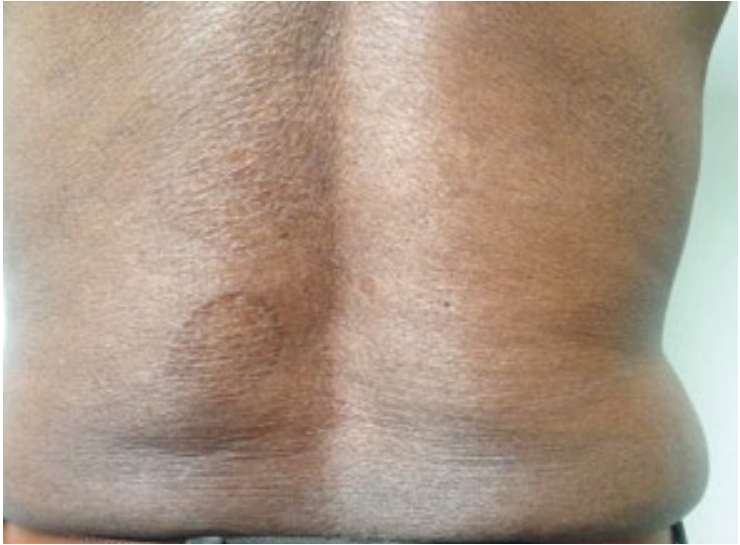

FIGURA 1. Placas circinadas con descamación ictiosiforme en la región dorso-lumbar

\section{CASO CLÍNICO}

Se trata de un hombre de 75 años de edad con fototipo VI, que consultó por un cuadro clínico de dos años de evolución caracterizado por unas lesiones pruriginosas en la piel de la región dorsal y lumbar que fueron aumentando de tamaño progresivamente, y que no había recibido tratamiento para las mismas. En la revisión por sistemas, no hubo sintomatología asociada, y los antecedentes médicos personales y familiares fueron negativos.

En el examen físico se encontraron, placas eucrómicas de bordes regulares con una forma circular geométrica muy bien definida en la piel de la región dorsolumbar, con presencia de descamación ictiosiforme (figuras 1 y 2). Se tomó una biopsia de piel en la que se reportó una epidermis con pigmentación uniforme de la capa basal por melanina, asociada a hiperqueratosis ortoqueratósica con ligera acantosis, hipogranulosis y zonas de ausencia de la capa granulosa y, en la dermis superficial, un ligero infiltrado linfocitario perifolicular que correspondía con el diagnóstico clínico de pitiriasis rotunda (figura 3).

Ante dicho diagnóstico, se decidió practicar algunos estudios de extensión, como ecografía hepatobiliar, tomografía axial computadorizada de abdomen, radiografía de tórax, hemograma, pruebas de función renal y hepática, pruebas para hepatitis B y hepatitis C, y prueba de Mantoux, sin hallazgos importantes. Sin haberse encontrado ninguna de las enfermedades sistémicas descritas asociadas, se inició tratamiento tópico con ácido retinoico al o,05 $\%$ en crema y urea al $10 \%$ en crema, sin haberse obtenido una reacción terapéutica adecuada a los seis meses de seguimiento.

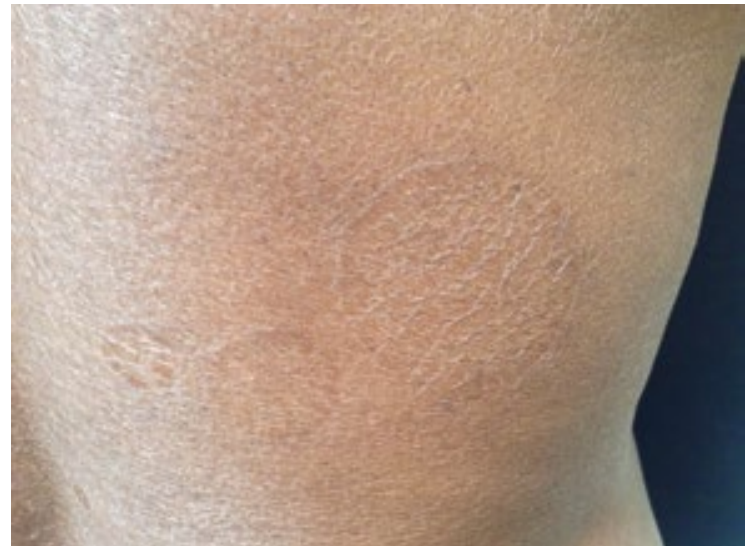

FIGURA 2. Acercamiento

\section{COMENTARIO}

La pitiriasis rotunda es una enfermedad rara de patogénesis desconocida, que usualmente se considera idiopática, aunque puede presentarse como parte de un síndrome paraneoplásico o asociada a enfermedades infecciosas sistémicas ${ }^{1-3}$. Se ha descrito principalmente en África, Italia y Japón; los reportes en población latinoamericana son escasos y usualmente se presenta en pacientes con fototipos oscuros. Se ha descrito que la enfermedad está relacionada con la disminución de algunas de las proteínas que intervienen en la formación de la envoltura celular 'cornificada', como la filagrina y la loricrina.

Recientemente, se ha descrito una disminución en la proteína filagrina-2, perteneciente a la familia de la proteína S1oo de tipo fusión, que usualmente se encuentra en la capa granulosa y córnea de la epidermis normal, hallazgos similares a los encontrados en enfermedades como la dermatitis atópica, la ictiosis vulgar y la psoriasis. Con estos hallazgos se ha propuesto que la enfermedad surge de una alteración en la diferenciación terminal de los queratinocitos y no de una disfunción de cada una de estas proteínas ${ }^{4}$.

Clínicamente es asintomática y se caracteriza por múltiples placas hipercrómicas o hipocrómicas, con descamación ictiosiforme y morfología circular geométricamente perfecta, que usualmente se encuentran en el tronco pero que pueden aparecer también en los brazos, glúteos y muslos 3.5 . Se propone una clasificación en la cual la de tipo I se presenta en población negra o asiática, con menos de 30 lesiones y asociada a enfermedades sistémicas, y la de tipo II se encuentra en pacientes blancos, con más de 30 lesiones, no se relaciona con enfermedades sistémicas y es familiar ${ }^{6}$. 


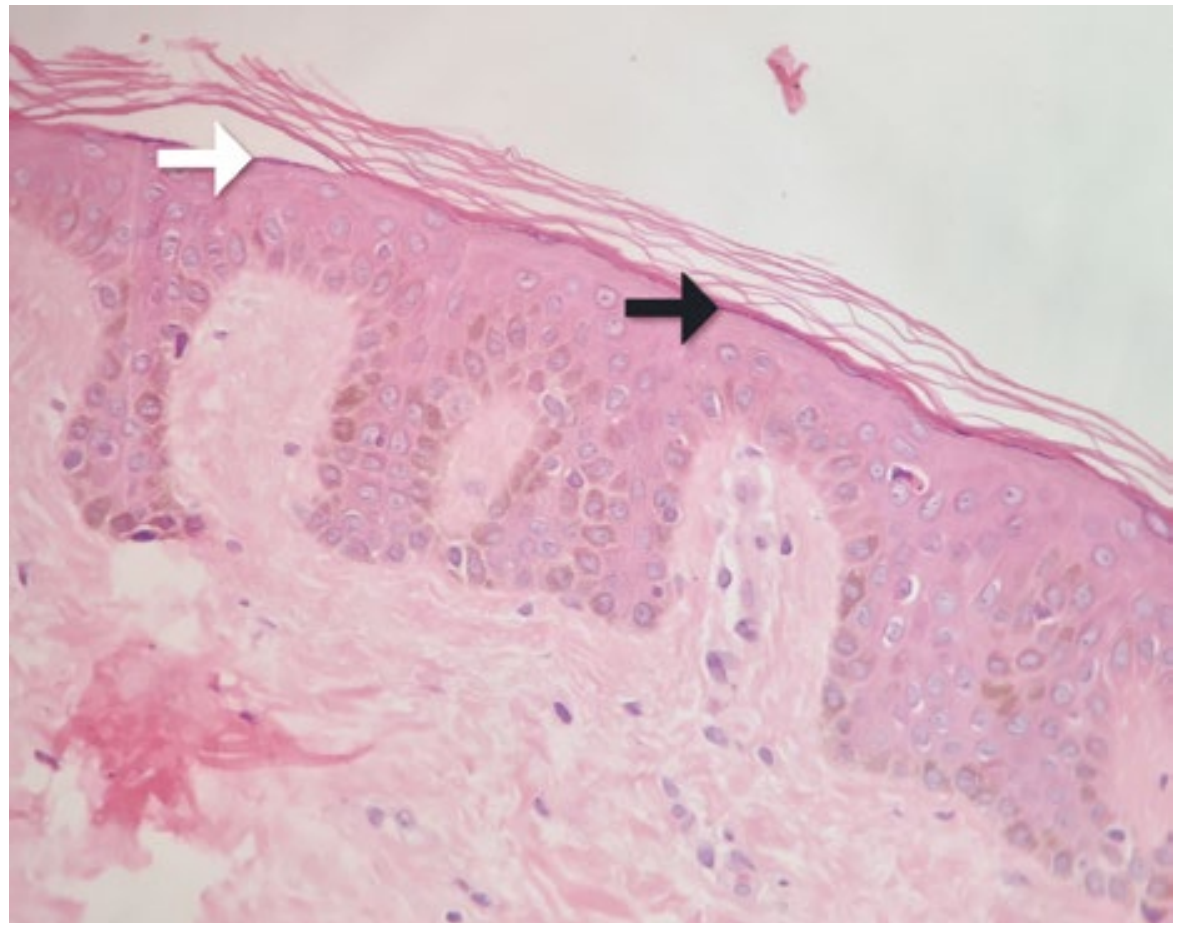

FIGURA 3. Pigmentación uniforme de la capa basal, hiperqueratosis ortoqueratósica, hipogranulosis (flecha negra) y agranulosis focal (flecha blanca).

Hematoxilina y eosina, $40 \mathrm{X}$
Se presenta con periodos de exacerbación y remisión, tanto en hombres como mujeres, con cierto predominio por los fototipos oscuros, en personas entre los 20 y los 45 años. Se han reportado casos de remisión de la dermatosis con la resección del tumor ${ }^{3}$.

En la histopatología se observa hiperqueratosis, ortoqueratosis, acantosis regional, con ausencia o reducción de la capa granular e hiperpigmentación de la capa basal debido al fototipo en el que usualmente se ha descrito ${ }^{1,2,3}$.

Las enfermedades malignas a las cuales se asocia son: adenocarcinoma de próstata, carcinoma del paladar, esofágico, hepatocelular y gástrico, leucemia linfoide crónica y mieloma múltiple ${ }^{7}$. En cuanto a las enfermedades infecciosas asociadas, se encuentran las del sistema urogenital (mujeres), las pulmonares, las hepáticas, la tuberculosis y la lepra ${ }^{7}$. Entre los diagnósticos diferenciales se debe considerar la tiña versicolor, el eritrasma, la lepra, la erupción fija, la pitiriasis alba y la pitiriasis rosada 5 . En cuanto al tratamiento, se han descrito los tópicos con retinoides, ácido salicílico y ácido láctico, sin conocerse su efectividad en estudios controlados, debido a la rareza de la enfermedad ${ }^{2,7}$.

\section{CONCLUSIÓN}

La pitiriasis rotunda es un trastorno de la queratinización con descamación ictiosiforme y disposición circular geométrica claramente distintiva, la cual se ha asociado a enfermedades subyacentes, incluso con síndrome paraneoplásico. Se presenta el caso de un paciente con esta enfermedad, quien -hasta el momento- no tiene una enfermedad sistémica asociada y se encuentra en seguimiento, ya que no se ha descrito cómo se comportan las asociaciones en temporalidad respecto a la aparición de esta enfermedad. Se recalca la necesidad del conocimiento de esta efermedad debido a las importantes entidades descritas asociadas y a su muy baja prevalencia en nuestro medio.

\section{REFERENCIAS}

1. Grimalt R, Gelmetti C, Brusasco A, Tadini G, Caputo R. Pityriasis rotunda: Report of a familial occurrence and review of the literature. J Am Acad Dermatol 1994;31:866-71.

2. Zur RL, Shapero J, Shapero H. Pityriasis rotunda diagnosed in Canada: Case presentation and review of the literature. J Cutan Med Surg. 2013;17:426-8.

3. Hasson I, Shah P. Pityriasis rotunda. Indian J Dermatol Venereol Leprol. 2003;69:50-1.

4. Makino T, Mizawa M, Seki Y, Hayashi M, Shimizu T. Decreased filaggrin-2 expression in the epidermis in a case of pityriasis rotunda. Clin Exp Dermatol. 2016;41:215-7.

5. Bakry OA, Samaka RM. Photoletter to the editor: Pityriasis rotunda. J Dermatol Case Rep. 2012;6:90-2.

6. Ena P, Cerimele D. Pityriasis rotunda in childhood. Pediatr Dermatol. 2002;19:200-3.

7. Ramos-E-Silva M, Carvalho JC, Carneiro SC. Cutaneous paraneoplasia. Clin Dermatol. 2011;29:541-7. 